\title{
ON A CHARACTERIZATION OF HILBERTIAN NORMS
}

\author{
BY
}

\author{
TEODOR PRECUPANU
}

\begin{abstract}
In this paper we prove that a characterization of hilbertian norms established in smooth normed spaces $X$ with $\operatorname{dim} X \geq 3$ also holds in two dimensional spaces.
\end{abstract}

Mathematics Subject Classification 2000: 46B20, 46C15.

Key words: smooth linear normed space, hilbertian norm, height of a triangle.

\section{Introduction}

Let $X$ be a real smooth linear normed space, that is there exists

$$
\lim _{t \rightarrow 0} \frac{\|x+t y\|^{2}-\|x\|^{2}}{2 t}
$$

for all $x, y \in X$. Usually, this limit is denoted by $n^{\prime}(x ; y)$. It is well known, that $n(x ; \cdot)$ is a linear continuous functional on $X$ and $n^{\prime}(x ; x)=\|x\|^{2}$ for every $x \in X$. Therefore, $X$ is a smooth space (see [1], [2]). Using an euclidean property of heights of a triangle, in [4] it is established the following characterization of hilbertian norms:

Theorem 1. In a smooth real linear normed space $X$ with $\operatorname{dim} X \geq 3$ the norm is hilbertian if and only

$$
\begin{aligned}
& n^{\prime}(y ; x)\left[n^{\prime}(x ; y) n^{\prime}(x-y ; x)-\|x\|^{2} n^{\prime}(x-y ; y)\right] \\
& =n^{\prime}(x ; y)\left[\|y\|^{2} n^{\prime}(x-y, x)-n^{\prime}(y ; x) n^{\prime}(x-y ; y)\right], \forall x, y \in X .
\end{aligned}
$$


In the present paper we show that this result is also true if $\operatorname{dim} X=2$. Our goals is to find the smooth norms of a two dimensional space $X$ which have the property (1). For this we use an idea of [3] taking into account that $n^{\prime}(x ; \cdot)$ is a linear functional for any $x \in X$. Thus, if $x=\left(x_{1}, x_{2}\right)$, $y=\left(y_{1}, y_{2}\right), x_{1}, x_{2}, y_{1}, y_{2} \in \mathbb{R}$, then

$$
n^{\prime}(x ; y)=A\left(x_{1}, x_{2}\right) y_{1}+B\left(x_{1}, x_{2}\right) y_{2},
$$

and

$$
\|x\|^{2}=A\left(x_{1}, x_{2}\right) x_{1}+B\left(x_{1}, x_{2}\right) x_{2},
$$

where $A, B$ are two real function on $\mathbb{R}^{2}$. Moreover, since $n^{\prime}(t x ; y)=t n^{\prime}(x ; y)$, for all $t \in \mathbb{R}$, we also have

$$
\begin{aligned}
& A\left(t x_{1}, t x_{2}\right)=t A\left(x_{1}, x_{2}\right), \\
& B\left(t x_{1}, t x_{2}\right)=t B\left(x_{1}, x_{2}\right), \quad \text { for all } t \in \mathbb{R} .
\end{aligned}
$$

\section{Proof of Theorem 1 in two dimensional spaces}

Taking into account the equalities (2) and (3), the property (1) becomes

$$
\begin{aligned}
& \left(A\left(y_{1}, y_{2}\right) x_{1}+B\left(y_{1}, y_{2}\right) x_{2}\right)\left[\left(A\left(x_{1}, x_{2}\right) y_{1}+B\left(x_{1}, x_{2}\right) y_{2}\right)\right. \\
& \left(A\left(x_{1}-y_{1}, x_{2}-y_{2}\right) x_{1}+B\left(x_{1}-y_{1}, x_{2}-y_{2}\right) x_{2}\right)-\left(A\left(x_{1}, x_{2}\right) x_{1}\right. \\
& \left.\left.+B\left(x_{1}, x_{2}\right) x_{2}\right)\left(A\left(x_{1}-y_{1}, x_{2}-y_{2}\right) y_{1}+B\left(x_{1}-y_{1}, x_{2}-y_{2}\right) y_{2}\right)\right] \\
& =\left(A\left(x_{1}, x_{2}\right) y_{1}+B\left(x_{1}, x_{2}\right) y_{2}\right)\left[\left(A\left(y_{1}, y_{2}\right) y_{1}+B\left(y_{1}, y_{2}\right) y_{2}\right)\right. \\
& \left(A\left(x_{1}-y_{1}, x_{2}-y_{2}\right) x_{1}+B\left(x_{1}-y_{1}, x_{2}-y_{2}\right) x_{2}\right)-\left(A\left(y_{1}, y_{2}\right) x_{1}\right. \\
& \left.\left.+B\left(y_{1}, y_{2}\right) x_{2}\right)\left(A\left(x_{1}-y_{1}, x_{2}-y_{2}\right) y_{1}+B\left(x_{1}-y_{1}, x_{2}-y_{2}\right) y_{2}\right)\right] .
\end{aligned}
$$

If $x=(1, t), y=(0, t), t \in \mathbb{R}$, we obtain

$$
\begin{aligned}
& (A(0, t)+t B(0, t))[t B(1, t)(A(1,0)+t B(1,0))-t B(1,0)(A(1, t)+t B(1, t))] \\
& =t B(1, t)[t B(0, t)(A(1,0)+t B(1,0))-t B(1,0)(A(0, t)+t B(0, t))]
\end{aligned}
$$

By (4) we get

$$
\begin{aligned}
& (A(0,1)+t B(0,1))[B(1, t)(A(1,0)+t B(1,0))-B(1,0)(A(1, t)+t B(1, t))] \\
& =t B(1, t)[B(0,1)(A(1,0)+t B(1,0))-B(1,0)(A(0,1)+t B(0,1))]
\end{aligned}
$$


3

that is,

$$
\begin{aligned}
& (A(0,1)+t B(0,1))(A(1,0) B(1, t)-B(1,0) A(1, t)) \\
& =t B(1, t)(A(1,0) B(0,1)-A(0,1) B(1,0)) .
\end{aligned}
$$

Therefore, if $B(1, t) \neq 0$, we obtain

$$
\frac{A(1, t)}{B(1, t)}=\frac{A(0,1)(t B(1,0)+A(1,0))}{B(1,0)(t B(0,1)+A(0,1))}
$$

whenever $t \neq-\frac{A(0,1)}{B(0,1)}, B(0,1) \neq 0$. Hence, the function $t \rightarrow \frac{A(1, t)}{B(1, t)}, t \in \mathbb{R}$, is a real function of homographic type, i.e.

$$
\frac{A(1, t)}{B(1, t)}=\frac{\alpha t+\beta}{\gamma t+\alpha}, \text { if } B(1, t) \neq 0, t \neq-\frac{\alpha}{\gamma},
$$

where $\alpha=A(0,1) A(1,0), \beta=A(0,1) B(1,0), \gamma=B(0,1) B(1,0)$.

On the other hand if $x_{1} \neq 0, x_{2} \neq 0$ and $B\left(1, \frac{x_{2}}{x_{1}}\right) \neq 0$ we have

$n^{\prime}(x, y)=x_{1} B\left(1, \frac{x_{2}}{x_{1}}\right)\left[\frac{A\left(1, \frac{x_{2}}{x_{1}}\right)}{B\left(1, \frac{x_{2}}{x_{1}}\right)} y_{1}+y_{2}\right]=x_{1} B\left(1, \frac{x_{2}}{x_{1}}\right)\left[\frac{\alpha x_{2}+\beta x_{1}}{\gamma x_{2}+\alpha x_{1}} y_{1}+y_{2}\right]$.

By (3) it follows

$$
\|x\|^{2}=x_{1} B\left(1, \frac{x_{2}}{x_{1}}\right) \frac{\beta x_{1}^{2}+2 \alpha x_{1} x_{2}+\gamma x_{2}^{2}}{\alpha x_{1}+\gamma x_{2}} .
$$

Since the norm is differentiable it is well known that

$$
A\left(x_{1}, x_{2}\right)=\frac{\partial}{\partial x_{1}}\left(\frac{1}{2}\|x\|^{2}\right), B\left(x_{1}, x_{2}\right)=\frac{\partial}{\partial x_{2}}\left(\frac{1}{2}\|x\|^{2}\right),
$$

and so, denoting $B(1, t)=f(t), t \in \mathbb{R}$, by usual computations we find

$$
\begin{aligned}
x_{1} f\left(\frac{x_{2}}{x_{1}}\right) & =\frac{\partial}{\partial x_{2}}\left(\frac{1}{2}\|x\|^{2}\right)=\frac{1}{2} x_{1} f\left(\frac{x_{2}}{x_{1}}\right) \frac{\left(2 \alpha^{2}-\beta \gamma\right) x_{1}^{2}+2 \alpha \gamma x_{1} x_{2}+\gamma^{2} x_{2}^{2}}{\left(\alpha x_{1}+\gamma x_{2}\right)^{2}} \\
& +\frac{1}{2} f^{\prime}\left(\frac{x_{2}}{x_{1}}\right) \frac{\beta x_{1}^{2}+2 \alpha x_{1} x_{2}+\gamma x_{2}^{2}}{\alpha x_{1}+\gamma x_{2}}
\end{aligned}
$$


i.e.

$$
\begin{aligned}
& x_{1} f\left(\frac{x_{2}}{x_{1}}\right) \frac{2\left(\alpha x_{1}+\gamma x_{2}\right)^{2}-\left(2 \alpha^{2}-\beta \gamma\right) x_{1}^{2}-2 \alpha \gamma x_{1} x_{2}-\gamma x_{2}^{2}}{\alpha x_{1}+\gamma x_{2}} \\
& =f^{\prime}\left(\frac{x_{2}}{x_{1}}\right)\left(\beta x_{1}^{2}+2 \alpha x_{1} x_{2}+\gamma x_{2}^{2}\right) .
\end{aligned}
$$

Dividing by $\beta x_{1}^{2}+2 \alpha x_{1} x_{2}+\gamma x_{2}^{2}$ we obtain the following simple differential equation $\frac{f^{\prime}(t)}{f(t)}=\frac{\gamma}{\gamma t+\alpha}$. Thus, it follows that $f(t)=(\gamma t+\alpha) k$, where $k$ is a real constant. In fact, $k=\|(1,0)\|^{-2}$.

Now, we return to the relation (6) and so we get

$$
\|x\|^{2}=k\left(\beta x_{1}^{2}+2 \alpha x_{1} x_{2}+\gamma x_{2}^{2}\right)
$$

If $B(1, t)=0$, then from (3) it follows that $\|(1, t)\|^{2}=A(1, t)>0$. In this case we consider the function $g(t)=A(1, t), t \in \mathbb{R}$. Similarly, we obtain the same equality (7). Also, since the norm is a continuous function, the equality (7) also holds if $x_{1}=0$ or $x_{2}=0$ or $\frac{x_{2}}{x_{1}}=-\frac{\alpha}{\gamma}$.

Finally, we established (7) for all $\left(x_{1}, x_{2}\right) \in \mathbb{R}^{2}$.

Therefore, the norm is obviously hilbertian.

Remark. Since a norm is hilbertian if its restriction to every two dimensional linear subspace is hilbertian, the above proof can be considered as a new proof of Theorem 4 in [4] for any linear normed space.

\section{REFERENCES}

1. Barbu, V.; Precupanu, T. - Convexity and Optimization in Banach Spaces, D. Reidel Publ. Comp., Dordrecht, 1986.

2. Holmes, R.B.--Geometric Functional Analysis and Its Applications, Graduate Texts in Mathematics, Springer-Verlag, New York-Heidelberg-Berlin, 1975.

3. Precupanu, T. - Ortogonality in linear spaces, (in Romanian), St. Cerc. Mat., 6 (1972), 881-889.

4. Precupanu, T.; Ionica, I. - Heights of a triangle in a linear normed space and Hilbertian norms, An. Ştiinţ. Univ. "Al.I. Cuza" Iaşi. Mat. (N.S.), 55 (2009), 35-47. 
5 